\title{
Clinicopathologic characteristics of Weneger's granulomatosis: A report of three cases
}

\author{
LI-YONG GAO, JIN DING and LI ZHAO
}

Department of Pathology, The First People's Hospital of Xuzhou, Xuzhou, Jiangsu 221002, P.R. China

Received July 22, 2015; Accepted February 3, 2016

DOI: $10.3892 /$ etm.2016.3046

\begin{abstract}
Weneger's granulomatosis (WG) is a rare autoimmune disease affecting a number of organs, including lungs and kidney. Although all age groups may be affected, the peak incidence occurs for individuals aged 30-50 years, with a slightly increased prevalence in males. In the current study, we present three cases of WG to describe clinical and pathological characteristics of this disease. Three patients with WG were assessed for clinical and pathological characteristics, and typical morphological findings using computerized tomography. Clinical manifestations included vasculitis, tissue necrosis, and the formation of granuloma. Following a large dose of glucocorticoid hormone treatment, the symptoms were successfully relieved in all three patients. In conclusion, the diagnosis of WG is dependent on pathological examination in combination with appropriate clinical and imaging data, and immunostaining with anti-neutrophil cytoplasmic antibody.
\end{abstract}

\section{Introduction}

Wegener's granulomatosis (WG) is a necrotizing granulomatous vasculitis that affects small arteries, veins and capillaries. Occasionally, large arteries in the upper and lower respiratory tract, and kidney are also involved. The joints, eyes and skin may also be affected in certain cases (1).

The disease was described for the first time in 1931 (2). Subsequently, Wegener et al described the disease in a more comprehensive manner (3). In that study, the disease was first described as a systemic, septic vascular disease (3). However, the cause of this disease is unknown. The annual incidence is $0.4 / 10$ million people. WG affects all age groups, with the peak incidence in individuals aged 30-50 years. The prevalence is slightly elevated in males (4). Previously, the average survival time of untreated Weneger's granulomatosis was 5 months,

Correspondence to: Dr Li-Yong Gao, Department of Pathology, The First People's Hospital of Xuzhou, 19 Zhongshan North Road, Xuzhou, Jiangsu 221002, P.R. China

E-mail: pathology163@163.com

Key words: Weneger's granulomatosis, characteristics, differential diagnosis with $80 \%$ of the patients succumbing to respiratory or renal failure within a year. Additionally, $>90 \%$ of the patients succumbed to the disease within two years (5). At present the majority of patients can have long-term relief under proper treatment.

The disease is divided into systemic and limited forms, depending on clinical manifestations (6). WG does not have unique symptoms. General symptoms include fever, fatigue, and anemia. Specific symptoms vary depending on the involved organ. Thus, the disease may manifest in the upper respiratory tract with runny nose, nose bleeds, nasal mucosa ulcers and nasal scab. Lower airway symptoms include chest tightness, cough, and hemoptysis. Kidney involvement manifests with proteinuria, hematuria, or renal insufficiency. Ocular manifestations include blurred vision and decreased visual acuity. Affected joints are marked by joint pain.

Due to its non-specific symptoms, WG is often underrecognized or misdiagnosed in the clinic. In the current study, we present three cases of WG to describe clinical and pathological characteristics in order to improve the identification of WG and reduce diagnostic errors.

\section{Clinical cases}

Ethics approval. Approval for the present study was obtained from The First People's Hospital of Xuzhou (Jiangsu, China).

Case 1. A 40-year-old male patient presented to The First People's Hospital of Xuzhou (Jiangsu, China) with a high temperature, cough, and hemoptysis that lasted for $>1$ month prior to admission to the Hospital. The patient was previously diagnosed with tuberculous pleurisy in another hospital a year earlier and received anti-tuberculosis drugs for 8 months. Following admission, the patient was found to have a temperature of $39.2^{\circ} \mathrm{C}$. Computerized tomography $(\mathrm{CT})$ was performed, which revealed multiple pulmonary nodules and irregular patchy shadows in both lungs. The laboratory examination demonstrated positive serum anti-neutrophil cytoplasmic and negative perinuclear (c- and p-ANCA, respectively) staining.

A percutaneous CT-guided lung biopsy was obtained. The biopsy specimen was fixed with $10 \%$ formalin and paraffin-embedded. Sections $(4 \mu \mathrm{m})$ were stained with hematoxylin and eosin staining Maixin Biotechnology Co., Ltd. (Shanghai, China) and observed under a light microscope Olympus CH30 (Tokyo, Japan). Parallel slides were stained 


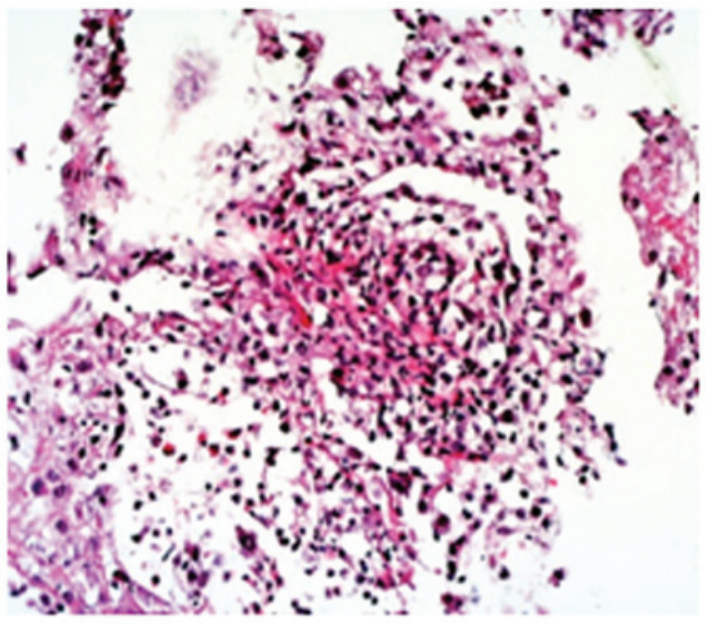

Figure 1. Alveolar septal vascular occlusion, neutrophil-dominated inflammation.

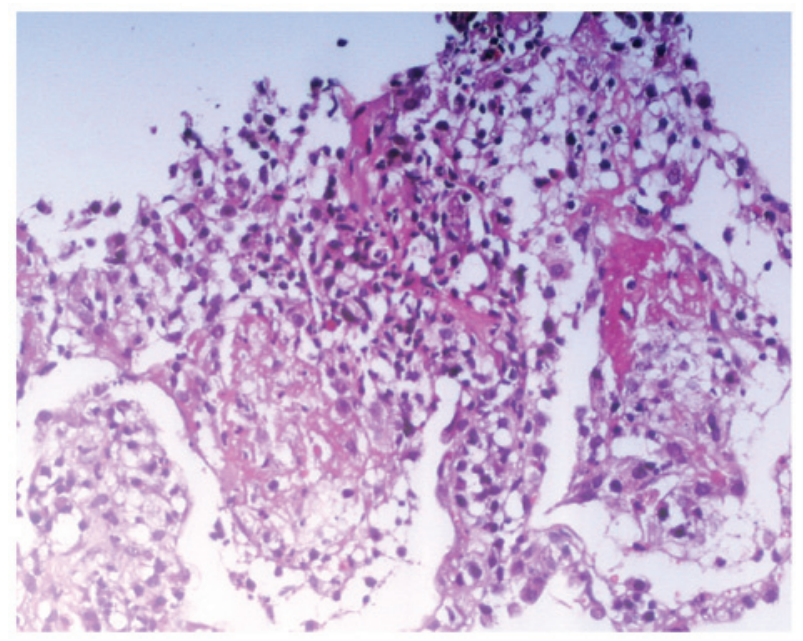

Figure 2. Formation of fibrinoid necrosis.

with periodic acid-Schiff and acid fast staining, and evaluated under a microscope.

The lung biopsy yielded two blocks of $1 \times 0.1 \times 0.1 \mathrm{~cm}$ of gray-white tissue. Microscopically, vascular cavity of the alveolar septa showed stenosis and occlusion. Neutrophils and some eosinophils infiltrated the vessel wall and adjacent region. Visible lymphoplasmacytic scattered infiltration and red dye fibrinoid deposits were evident. Unconspicuous granuloma formed by few multinucleated giant cells with an organized structure were identified in certain parts of the region (Figs. 1-3). Thus, pathological diagnosis complied with the diagnosis of ANCA-associated vasculitis (i.e., WG).

Case 2. A 66-year-old male patient reported having an intermittent cough for 3 years. Over a month prior to admission to the Hospital, the cough became hoarse. The patient's body temperature was normal when he presented to the hospital. The patient was laryngoscopically examined by an ENT specialist owing to the hoarseness. A subglottic cauliflower neoformation with proneness to hemorrhaging was observed. The glottis was narrow. The vocal cord abduction was limited. A biopsy

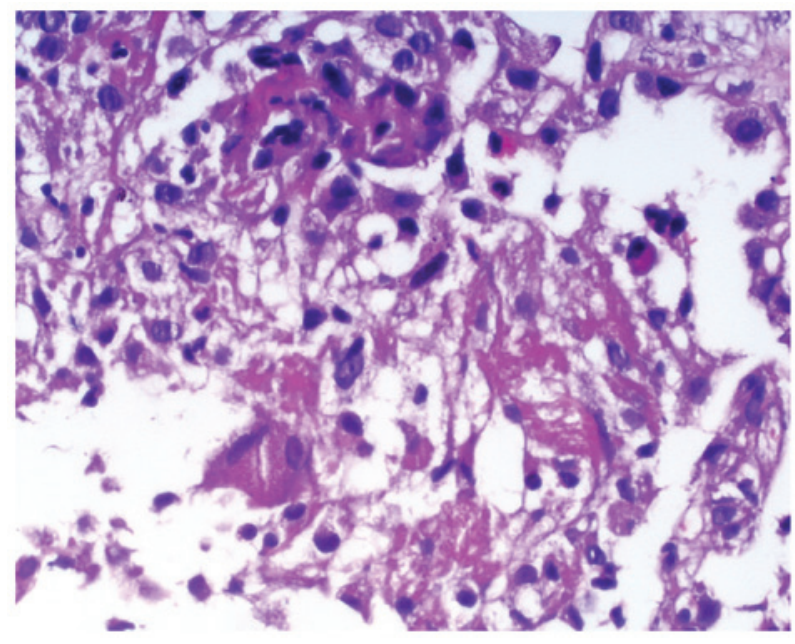

Figure 3. Granuloma formed by multinucleated giant cells and tissue cells.

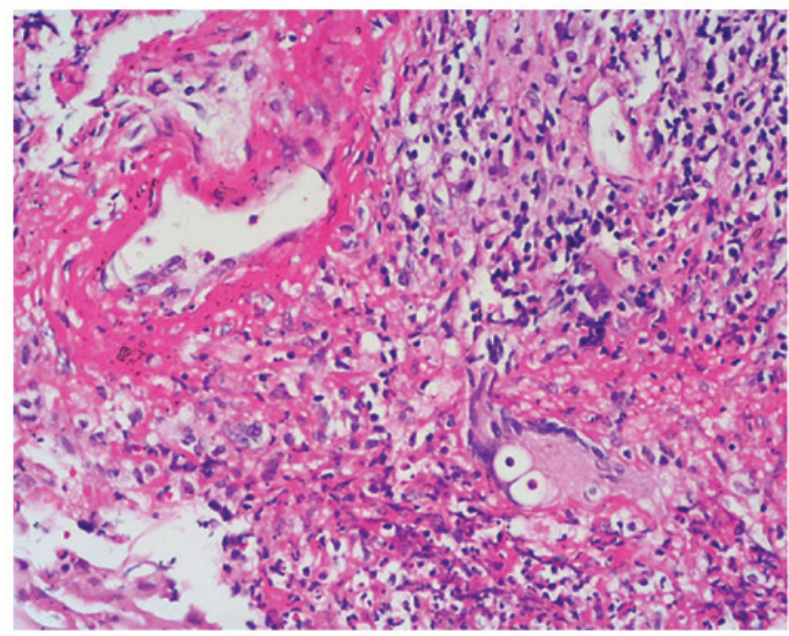

Figure 4. Fibrinoid necrosis of vascular wall, partial tissue necrosis, visible multinucleated giant cells, and infiltration of neutrophils.

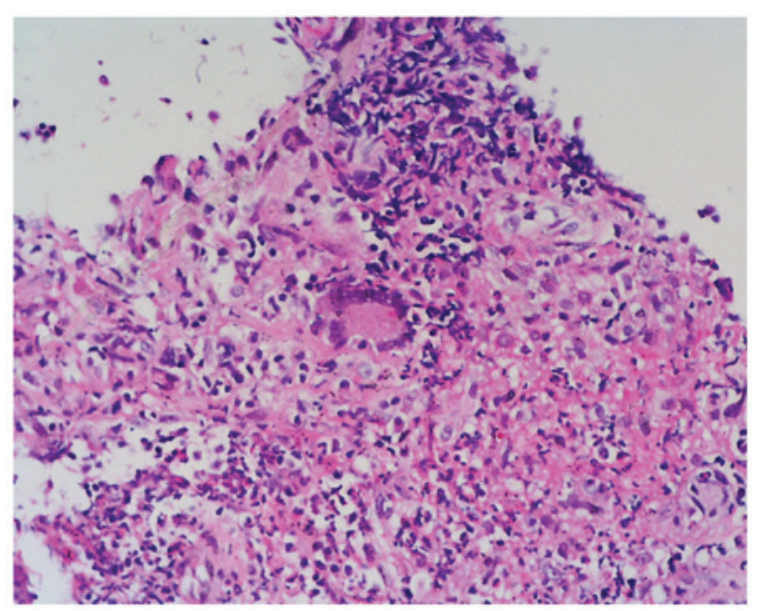

Figure 5. Tissue necrosis. Giant cells are evident. Some fibrin exudation and infiltration of neutrophils is evident.

was performed and assessed for laryngeal carcinoma. The CT revealed a number of neoformations on the right nasal and 


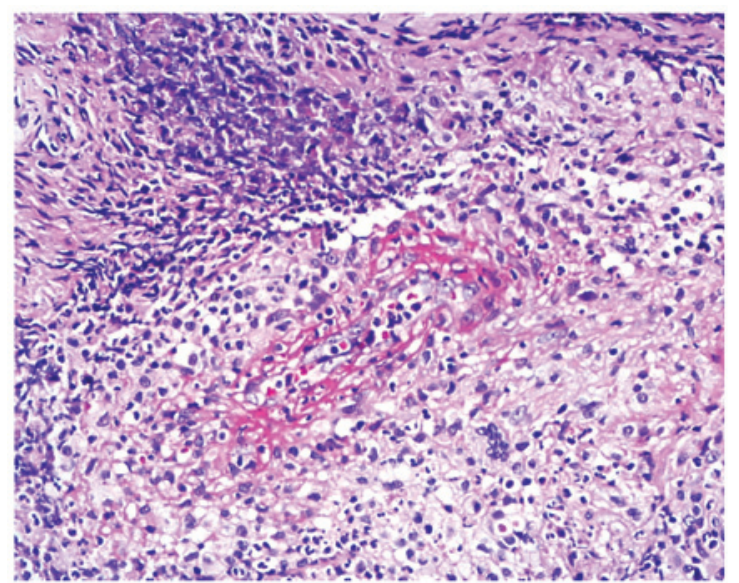

Figure 6. Fibrinoid necrosis of the vascular wall, infiltration of neutrophils, few multinucleated giant cells, visible basophilic necrosis.

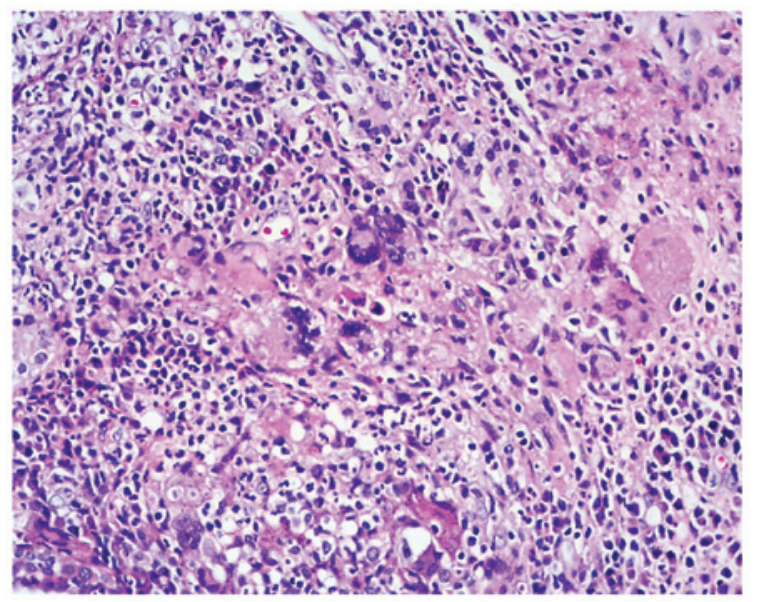

Figure 7. Multinucleated giant cell aggregation, and granuloma formation. Lymphocytes and plasma cells are evident. Infiltration of neutrophils.

maxillary sinuses, and multiple pulmonary occupying lesions. A nasal neoplasm biopsy was obtained by an ENT specialist. The laboratory examination revealed positive serum $\mathrm{p}$ - and negative c-ANCA.

Gross examination of the biopsy of the glottis revealed a bean-sized mass of pale gray-red broken tissue. Microscopically, squamous epithelium showed hyperplasia in the superficial areas, partial disappearance of epithelium, visible necrosis, fibrin exudation, fibrinoid necrosis of parts of the vascular wall, and neutrophil infiltration. Small multinucleated giant cells were observed near the necrotic exudation (Figs. 4 and 5). Pathological diagnosis did not exclude WG. Gross examination of the nasal biopsy demonstrated a mass of ash-red and gray-white tissue with the size of $4 \times 2.5 \times 1 \mathrm{~cm}$. Some parts of the tissue were soft, while other parts flexible. Microscopically, there were numerous lymphocytes, plasma cells and neutrophils within the tissue. Fibrinoid necrosis was evident in part of the vascular wall. Necrotic tissue was basophilic and distributed in a patchy manner. Granuloma was formed by few multinucleated giant cells, and was scattered or focally distributed. Small suppurative foci formed in parts of the region (Figs. 6 and 7). Based on the

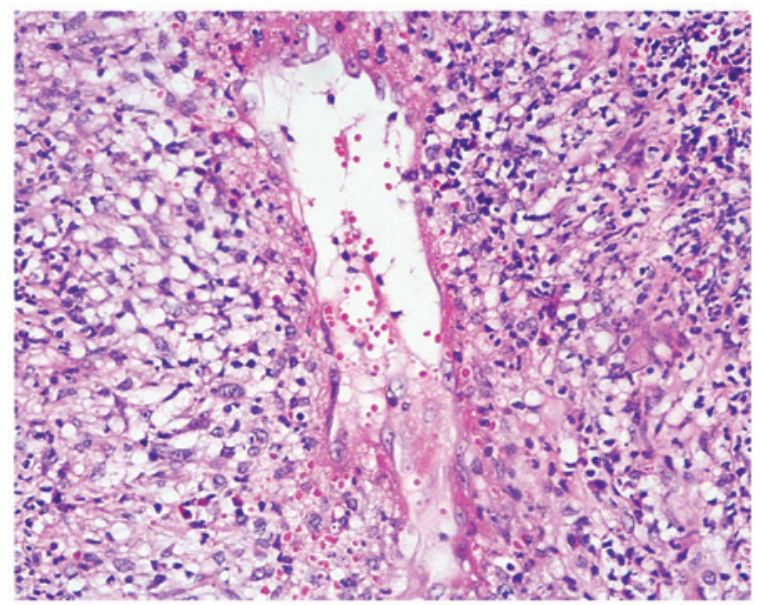

Figure 8. Fibrinoid necrosis of the vessel wall. Inflammatory cell infiltration.

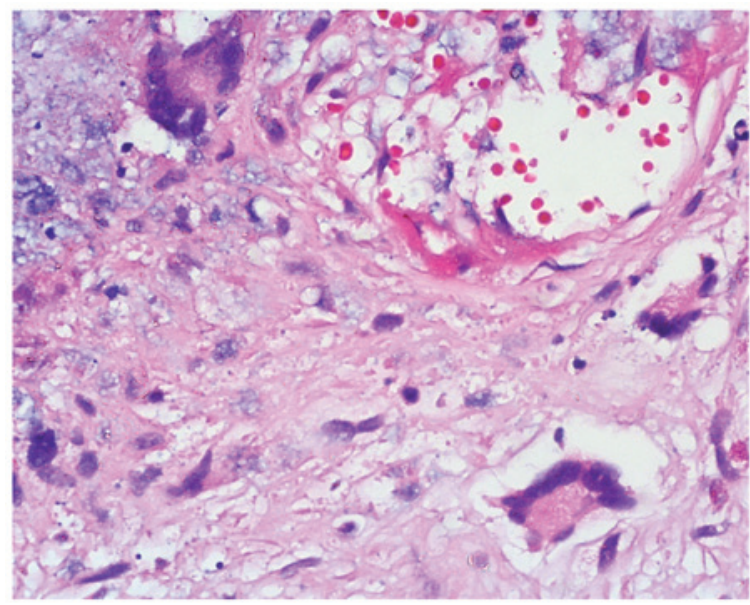

Figure 9. Formation of multinucleated giant cells around the necrotic region, and inflammatory cell infiltration.

pathological findings, a WG diagnosis was made which was confirmed by positive ANCA staining, and negative fungal PAS and acid-fast staining.

Case 3. A 26-year-old male patient experienced bilateral exophthalmos for $>6$ months prior to admission. Five days prior to admission, the symptoms in the right eye worsened. The CT revealed a tumor in the procuratorial right orbital area with unclear border and uniform texture. The patient was diagnosed with WG in another hospital and treated with prednisone (20 mg/day). The right eye proptosis was aggravated after common colds. The laboratory examination demonstrated negative serum $\mathrm{p}$ - and positive c-ANCA.

Gross examination of the right orbital tumor resection revealed a block of gray ash-red tissue with a sized of $3 \times 2.5 \times 1 \mathrm{~cm}$, as well as a gray and pinkish-gray section. The texture was flexible. Microscopically, there were lymphocytes, plasma cells and neutrophils infiltrating the tissue, including fat tissue and striated muscle. Part of the small vascular wall showed fibrinoid necrosis. Patchy necrosis was basophilic. Partial necrosis was distributed around the periphery of fibrinoid necrosis of small vessels. The typical granulation-like 
structure comprised multinucleated giant cells (Figs. 8 and 9). Pathological diagnosis confirmed WG.

Treatment and prognosis. In all three patients, symptoms were relieved following a large dose of hormone treatment. During the active phase with prednisone (Weihai Disu Pharmaceutical Co., Ltd., China), 1.0 to $1.5 \mathrm{mg}$ were administered once per day for 4-6 weeks. When the disease was relieved, we reduced prednisone dose and maintained in a small doses. The patients were discharged and followed up for 6 months. All three patients recovered well.

\section{Discussion}

WG is a rare autoimmune disease that mainly affects small vessels. Necrotizing vasculitis and granulomatous inflammation are basic pathological characteristics of this disease. WG is a multisystem disorder disease. Organ involvement defines clinical manifestations (7). The symptoms also include nasal and paranasal sinusitis, lung disease and progressive renal failure. The joints, eyes, and skin may also be affected in certain patients.

The causes of this disease remain to be determined. In the current study, three cases of WG were investigated to determine whether pathological examination in combination with appropriate clinical and imaging data would be useful to identify this disease in patients. The pathological examination showed typical tissue necrosis, vasculitis and the formation of granuloma structure. Typical chronic granulomatous inflammation performance was not necessarily obvious in biopsies, but manifested as a single scattering multinucleated giant cell or several multinucleated giant cells. Tissue necrosis was basophilic or fibrinoid, with infiltrating neutrophils, lymphocytes or plasma cells. The proliferation and edema of vascular endothelial cells caused luminal stenosis and occlusion. Vascular wall showed fibrinoid necrosis. Addtionally, it was found that due to extensive neutrophil aggregation, a small abscess may form.

The diagnostic standards of WG were proposed by the American Rheumatism Association in 1990 and include the following (8): i) Nasal or stomatitis symptoms (pain or painless oral ulcers, bloody nasal discharge); ii) microscopic hematuria (red blood cells $>5 /$ high power field) or red blood cell casts; iii) chest X-ray film reveals nodules, fixed infiltration, or cavity formation; and iv) biopsy shows granulomatous inflammation of arteries or arterioles, and surrounding tissue.

Two or more positive criteria indicates a diagnostic sensitivity of $88.2 \%$ and specificity of $92 \%$ (8).

Pathological examination is important for diagnosing WG. Serum ANCA examination and biopsy findings become increasingly important in the diagnosis of WG, especially in cases with no microscopic hematuria or red blood cell casts. The use of ANCA significantly increased diagnostic accuracy in WG7. However, an ANCA analysis has yet to be included in the diagnostic criteria of WG by the American Rheumatism Association (8). ANCA are heterologous antibodies against neutrophil cytoplasmic antigens (9). These antibodies are divided into cytoplasmic (c-ANCA), perinuclear (PANCA), and atypical (X-ANCA) types. Of these three types, c-ANCA are most commonly found in $\mathrm{WG}$, with the specificity of diagnosis reaching $95-98 \%(9,10)$. Fewer than $10 \%$ of the patients can also express p-ANCA, while some patients are ANCA-negative $(10,11)$.

Other diseases, such as the Churg-Strauss syndrome or microscopic polyangiitis, can also be associated with positive ANCA. Furthermore, the production of ANCA is associated with infection or treatment with certain drugs (12). Therefore, diagnosis of WG should include clinical manifestation, pathological diagnosis, laboratory tests, and imaging data.

A differential diagnosis should be performed with the following diseases: i) rhinitis, nasosinusitis: These diseases present with lymphocyte and plasma cell infiltration, and tissue edema. There is no vasculitis or multinucleated cell aggregation forming granuloma, and less tissue necrosis (13). ii) Nasal NK/T cell lymphoma: This is a type of lymphoma where lymphocytes have empty pale cytoplasm or distorted nucleus (atypical lymphocytes). It may present with substantial coagulation necrosis and infiltration of neoplastic lymphocytes to the vascular center (14). However, granulomas formed by multinucleated cells were not typically identified. iii) Tuberculosis is a disease that often appears with caseous necrosis, surrounded with giant cell of Langerhans, and the formation of large quantities of epithelioid cells (15). Typically, acid-fast staining is positive. iv) Lymphomatoid granulomatosis (16): This disease is associated with angiocentric and vascular damage of extranodal lymphoid tissue proliferation. Infiltrating cells are small lymphocytes, plasma cells, and large atypical lymphocytes. Granulomas and neutrophil infiltration are absent. Furthermore, this disease does not affect the upper respiratory tract. v) Microscopic polyangiitis (17): This is systemic necrotizing vasculitis involving small vessels. It often manifests with necrotizing glomerulonephritis and pulmonary capillary inflammation. Involvement of the upper respiratory tract and presence of pulmonary shadows are rare. vi) Allergic granulomatous angiitis (18): The disease often appears as pulmonary and extrapulmonary small artery, with vein inflammation and necrosis, and granulomas. There can be some eosinophil infiltration in peripheral blood and tissue lesions. Allergic granulomatous angiitisis often associated with the occurrence of asthma.

WG is most commonly treated with glucocorticoid hormones. Immunosuppressive agents may also be used. Prognosis in untreated WG is extremely poor. Over $90 \%$ of the patients die of respiratory failure and/or renal failure within two years. Early diagnosis of WG and timely treatment can effectively reduce the mortality rate, especially when a combination therapy of glucocorticoids with cyclophosphamide is administered. This therapy can lead to long-term remission.

In conclusion, the study on the three cases of WG has demonstrated that tissue necrosis, vasculitis, and the formation of granuloma are typical microscopic indicators of WG. Furthermore, fibrinoid necrosis of the vessel wall and surrounding tissue, and presence of multi-nucleated giant cells can also be indicative of WG.

\section{References}

1. Hoffman GS, Kerr GS, Leavitt RY, Hallahan CW, Lebovics RS, Travis WD, Rottem M and Fauci AS: Wegener granulomatosis: an analysis of 158 patients. Ann Intern Med 116: 488-498, 1992. 
2. Klinger H: Grenzformen der periarteritis nodosa. Frankf Z Pathol 42: 455-480, 1931 (In German).

3. Wegener F: Über generalisierte, septische Efäberkrankungen. Verh Dtsch Pathol Ges 29: 202-210, 1936 (In German).

4. Lu ZY and Zhong NS: Internal Medicine. People's Medical Publishing House, Beijing, pp884-885 2011.

5. Gadó K1, Z Szabó L, Csákó L and Domján G: Wegener's granulomatosis. Orv Hetil 154: 1083-1095, 2013.

6. Chen DY, Liang LQ and Zhan ZP: Limited and severe Wegener's granulomatosis: clinical features. Chin Remedies Clin 10: 489-492, 2010 (In Chinese).

7. Yu SY and Sun B: Clinical Diagnosis and Treatment of Respiratory Department of Internal Medicine. Union Medical College Press, Beijing, 2010.

8. Leavitt RY, Fauci AS, Bloch DA, Michel BA, Hunder GG Arend WP, Calabrese LH, Fries JF, Lie JT, Lightfoot RW Jr, et al: The American College of Rheumatology 1990 criteria for the classification of Wegener's granulomatosis. Arthritis Rheum 33: 1101-1107, 1990

9. Vassilopoulos D, Niles JL, Villa-Forte A, Arroliga AC, Sullivan EJ, Merkel PA and Hoffman GS: Prevalence of antineutrophil cytoplasmic antibodies in patients with various pulmonary diseases or multiorgan dysfunction. Arthritis Rheum 49: 151-155, 2003

10. Zak-Gołab A, Hrycek A, Holecki M and Chudek J: Diagnostic value of antineutophil cytoplasmic antibodies. Wiad Lek 64 37-42, 2011 (In Polish).
11. Chen M, Yu F, Zhang Y and Zhao MH: Antineutrophil cytoplasmic autoantibody-associated vasculitis in older patients. Medicine (Baltimore) 87: 203-209, 2008.

12. Kallenberg CG: Key advances in the clinical approach to ANCA-associated vasculitis. Nat Rev Rheumatol 10: 484-493, 2014.

13. Stierna P and Carlsöö B: Histopathological observations in chronic maxillary sinusitis. Acta Otolaryngol 110: 450-458, 1990.

14. Chubachi A, Imai H, Nishimura S, Saitoh M and Miura AB: Nasal T-cell lymphoma associated with hemophagocytic syndrome. Immunohistochemical and genotypic studies. Arch Pathol Lab Med 116: 1209-1212, 1992.

15. Sweany HC and Seiler HH: The pathology and bacteriology of resected lesions in pulmonary. Dis Chest 29: 119-152, 1956.

16. Feng RE, Liu HR, Liu TH, Chen J, Ling Q, Shi XH, Zhong DR, Luo YF and Cao JL: Pulmonary lymphomatoid granulomatosis: An immunohistochemical and gene rearrangement study. Zhonghua Bing Li Xue Za Zhi 40: 460-464, 2011 (In Chinese).

17. Chinese Medical Association of Rheumatology: Microscopic polyangiitis diagnosis and treatment guidelines. Chin J Rheumatol 15: 259-261, 2011 (In Chinese).

18. Jones RB, Tervaert JW, Hauser T, Luqmani R, Morgan MD, Peh CA, Savage CO, Segelmark M, Tesar V, van Paassen P, et al; European Vasculitis Study Group: Rituximab versus cyclophosphamide in ANCA-associated renal vasculitis. N Engl J Med 363: 211-220, 2010. 